\title{
Discordância na percepção da imagem corporal avaliada por meio da escala de silhuetas de forma crescente e aleatória
}

\author{
Divergences in the perception of body image evaluated by the \\ silhouette scale in ascending and random order
}

\author{
Ana Carolina Paludo'; Enio Ricardo Vaz Ronque2; Gabriela Blasquez;; Lidia Raquel Ferreira \\ Gonçalves"; Hélio Serassuelo Junior ${ }^{5}$ \\ ${ }^{1}$ Mestranda em Educação Física - PPGEF UEM/UEL. Londrina, PR - Brasil. \\ ${ }^{2}$ Doutor em Educação Física - Unicamp, Docente - UEL/CEFE. Londrina, PR - Brasil. \\ ${ }^{3}$ Mestre em Educação Física - PPGEF UEM/UEL, Docente - UNIRP. São José do Rio Preto, SP - Brasil \\ ${ }^{4}$ Graduada em Educação Física - UEL/CEFE. Londrina, PR - Brasil. \\ ${ }^{5}$ Doutor em Educação Física - USP, Docente - UEL/CEFE e Unopar. Londrina, PR - Brasil. \\ Endereço para correspondência \\ Hélio Serassuelo Júnior \\ 86051-980 - Londrina - PR [Brasil] \\ heliojr@onda.com.br
}

\begin{abstract}
Resumo
Introdução: Utilizar um instrumento que avalie adequadamente a insatisfação corporal pode permitir um diagnóstico e tratamento precoce de adolescentes. Objetivo: Verificar a possível concordância nos resultados da escala de silhuetas em formato crescente e aleatório. Métodos: Participaram do estudo 238 adolescentes do sexo feminino, com idade entre 15 e 17 anos. Avaliaram-se medidas de massa corporal e estatura para cálculo do índice de massa corporal. Aplicou-se a escala de silhueta proposta por Stunkard et al. ${ }^{7}$, de forma crescente e, posteriormente, de forma aleatória. Para isso, foram aplicados os testes de McNemar e o índice Kappa. Resultados: Houve diferenças significantes entre as duas formas de aplicar a escala de silhuetas $(\mathrm{p}<0,001)$ e a concordância entre as medidas foi moderada $(0,46)$. Conclusão: As formas de aplicação da escala de silhuetas apresentaram-se discordantes. Além disso, a aplicação da forma crescente parece influenciar as adolescentes em uma opção distorcida da sua própria imagem corporal.
\end{abstract}

Descritores: Adolescentes; Avaliação; Imagem corporal.

\begin{abstract}
Introduction: Using an instrument to properly assess body dissatisfaction may enable early diagnosis and treatment of adolescents. Objective: To investigate the possible convergence in results of the silhouette scale arranged in ascending and random order. Methods: The study included 238 female adolescents, aged between 15 and 17 years. Body weight and height measurements were taken for calculating the body mass index. The silhouette scale proposed by Stunkard et al. ${ }^{7}$ was applied first in ascending order and then in random order. For this, McNemar's test and the Kappa Index were used. Results: There were significant differences between the two ways of applying the silhouette scale $(p<0.001)$ and the convergence between measures was moderate (0.46). Conclusion: The manners of applying the silhouette scale showed divergences. Moreover, its application in ascending order seems to influence adolescents into adopting a distorted body image.
\end{abstract}

Key words: Adolescents; Body image; Assessment. 


\section{Introdução}

A imagem corporal, definida como a percepção que o indivíduo exerce do tamanho ou partes específicas do seu próprio corpo, têm-se tornado foco de diversos estudos nos últimos anos. Percebe-se que cada vez mais indivíduos, com diferentes características, apresentam-se com uma imagem corporal negativa de si mesmo, o que demonstra a insatisfação corporal crescente na sociedade ${ }^{1,2}$. Atrelada a essa insatisfação nota-se também o aumento nos casos de desordens alimentares e distúrbios psicológicos oriundos dessa dificuldade de adequação social, principalmente entre os adolescentes ${ }^{3,4}$, uma vez que a cobrança por padrões de beleza impostos pela mídia são fortes influenciadores nessa população ${ }^{5}$, além dos aspectos afetivos, cognitivos e descritivos ${ }^{6}$.

O estudo da imagem corporal é uma importante ferramenta para estratégias de prevenção e tratamento de distúrbios relacionados a uma imagem corporal negativa. Assim, métodos válidos são necessários para avaliação desse constructo. Existe uma variedade de medidas para avaliar os aspectos da imagem corporal, que compreende questionários e escalas de figuras ou silhuetas.

A escala de figuras ou silhuetas é comumente utilizada para determinar a insatisfação com o tamanho corporal, em que o formato do corpo é disposto em ordem crescente, variando de silhuetas muito finas a obesas ${ }^{7-10}$.

Uma das escalas mais utilizadas é a escala de silhuetas proposta por Stunkard et al. ${ }^{7}$ a qual consiste em nove silhuetas distribuídas em ordem crescente em relação à forma corporal. Essa escala já foi validada para utilização em diferentes populações e em diferentes países ${ }^{11,12,13}$, sempre disposta em seu formato original.

No entanto, a disposição das silhuetas na forma crescente pode provocar estimativas elevadas de insatisfação corporal ${ }^{14}$ e diferentes percepções sobre o formato corporal ${ }^{15}$. Assim, neste estudo, tem-se como objetivo verificar a concordância entre as silhuetas dispostas em ordem crescente e aleatória em adolescentes do sexo feminino.

\section{Metodologia}

Este estudo é caracterizado como descritivo, correlacional de delineamento transversal.

\section{Amostra}

A amostra foi composta por 238 adolescentes do sexo feminino, com idade entre 15 e 17 anos, cursando o segundo ano do ensino médio de seis escolas do Município de Marechal Cândido Rondon - Paraná.

Como critério de inclusão, as jovens deveriam estar matriculadas na escola avaliada, pertencer a série estabelecida e estar presente na aula de Educação Física no dia da avaliação.

As adolescentes foram informadas que a participação no estudo era voluntária e que todos os dados seriam confidenciais. Os diretores das escolas, assim como pais ou responsáveis, assinaram um termo de consentimento livre e esclarecido autorizando a participação das alunas na pesquisa.

\section{Instrumentos e procedimentos}

A coleta de dados ocorreu durante as aulas de Educação Física, e as adolescentes responderam os questionários, em uma sala de aula, com orientação coletiva dos pesquisadores e presença do professor responsável.

Foi utilizada a escala de silhueta proposta por Stunkard, Sorenson e Schlusinger ${ }^{7}$, a qual consiste em nove figuras que representam formas de contorno ou silhueta corporal dispostas em ordem crescente, abrangendo a silhueta bem magra (silhueta 1) até a obesa (silhueta 9) (Figura 1).

Após a visualização das figuras, as adolescentes responderam as seguintes perguntas contidas no questionário: qual a silhueta que melhor representa sua aparência física atual? Qual a silhueta que você gostaria de ter? A primeira 
pergunta refere-se a real forma corporal que o indivíduo se encontra, e a segunda pergunta está relacionada à forma de silhueta que a pessoa julga ser ideal. A insatisfação com a imagem corporal foi verificada por meio da discordância entre a silhueta real e a ideal, classificando as participantes em satisfeitas ou insatisfeitas.

Primeiramente, as adolescentes responderam o questionário no formato original proposto pelos autores ${ }^{7}$, com as silhuetas dispostas em forma crescente, seguindo as duas questões a respeito de seu formato real e ideal. Em um segundo momento, elas responderam o questionário das silhuetas de forma aleatória, mantendo as mesmas perguntas sobre a silhueta real e ideal.

Foram realizadas medidas antropométricas de massa corporal e estatura para caracterização da amostra, por meio de uma balança digital (Tanita), com resolução de $100 \mathrm{~g}$ e de um estadiômetro de parede, com precisão de 0,1 $\mathrm{cm}$, seguindo as recomendações de Gordon et al. ${ }^{16}$. Por meio dessas medidas foi calculado o índice de massa corporal (IMC = massa corporal/estatura ${ }^{2}$ ).

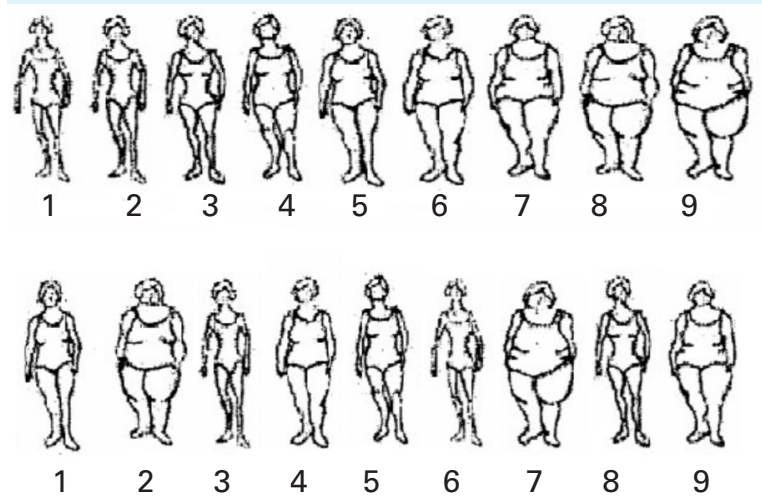

Figura l: Distribuição das silhuetas na forma crescente e aleatória, respectivamente

\section{Anólise estatística}

Para análise descritiva, os dados foram expressos em média e desvio-padrão para as variáveis numéricas, e a distribuição de frequências (absoluta e relativa) foi utilizada para descrever a variável categórica. O teste de McNemar foi aplicado para verificar as diferenças entre as proporções de adolescentes na classificação da percepção de imagem corporal, quando aplicado na forma crescente e na aleatória, e o índice Kappa foi empregado para verificar a concordância entre as proporções. A significância adotada foi $\mathrm{p}<0,05$, e os dados foram tratados no pacote estatístico SPSS 17.0.

\section{Resultados}

Os dados das características gerais da amostra estão descritos na Tabela 1. Observa-se que o IMC médio das adolescentes foi $21 \mathrm{~kg} / \mathrm{m}^{2}$, considerado como peso normal para a idade média dessas participantes.

Tabela l: Características gerais da amostra $(n=238)$

\begin{tabular}{ccc}
\hline Variável & $\begin{array}{c}\text { Média } \\
\text { (desvio-padrão) }\end{array}$ & Min.-Máx. \\
\hline Idade (anos) & $15,92(0,92)$ & $15-17$ \\
Massa corporal $(\mathrm{kg})$ & $57,38(10,87)$ & $40,50-114,9$ \\
Estatura $(\mathrm{cm})$ & $165,68(7,11)$ & $140-187$ \\
IMC $\left(\mathrm{kg} / \mathrm{m}^{2}\right)$ & $20,85(3,26)$ & $14,69-37,95$ \\
\hline
\end{tabular}

Na Figura 2, estão apresentadas as distribuições das frequências absolutas das (in) satisfação das adolescentes, quando aplicada a escala pela forma crescente e pela aleatória.

Verifica-se predomínio de insatisfação em relação à silhueta das voluntárias independentemente da forma de aplicação do instrumento. No entanto, a insatisfação com a própria silhueta é maior quando o questionário é aplicado na forma crescente $(\mathrm{n}=197,83 \%)$ do que na aleatória $(n=163,68 \%)$.

A Tabela 2 demonstra a frequência percentual de adolescentes (in) satisfeitas com a imagem corporal, quando avaliadas de forma aleatória e crescente.

O teste de McNemar mostrou diferenças significantes entre as duas formas de aplicar a escala de silhuetas, e o índice Kappa apontou fraca concordância entre as medidas. 


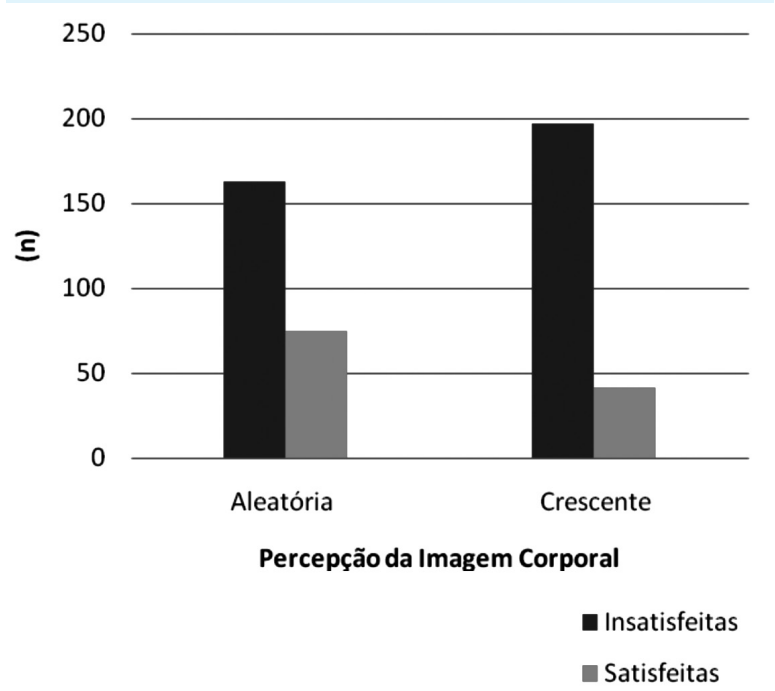

Figura 2: Distribuição das frequências absolutas de (in) satisfação por meio de escala de silhuetas de forma crescente e aleatória

Tabela 2: Concordância na classificação da percepção da imagem corporal, quando aplicado o instrumento de forma aleatória e crescente

\begin{tabular}{ccccc}
\hline $\begin{array}{c}\text { Escala de } \\
\text { silhueta } \\
\text { aleatória }\end{array}$ & $\begin{array}{c}\text { Escala de silhueta crescente } \\
\text { Satisfação } \\
(\%)\end{array}$ & $\begin{array}{c}\text { Insatisfação } \\
-(\%)\end{array}$ & $P$ & Kappa \\
\hline $\begin{array}{c}\text { Satisfação } \\
\text { (\%) }\end{array}$ & 14,3 & 17,2 & & \\
$\begin{array}{c}\text { Insatisfação } \\
(\%)\end{array}$ & 2,9 & 65,5 & 0,001 & 0,46 \\
\hline
\end{tabular}

\section{Discussão}

Neste estudo, analisou-se a percepção da imagem corporal por meio de um questionário em que as silhuetas foram dispostas de maneira crescente e aleatória. O principal achado deste trabalho consiste na discordância apresentada entre as duas formas de apresentação das silhuetas, ou seja, com cada instrumento, avaliou-se de forma distinta a percepção da imagem corporal das adolescentes.

A média do IMC das jovens estudadas foi $21 \mathrm{~kg} / \mathrm{m}^{2}$, classificando-as como peso normal, de acordo com a proposta de Cole et al. ${ }^{17}$, apesar disso, houve uma elevada prevalência de in- satisfação com a imagem corporal em $83 \%$ das participantes, quando analisada na sua forma original (crescente), mostrando prevalência semelhante com adolescentes de outros países ${ }^{18,19}$, avaliadas pelo mesmo instrumento. Entretanto, quando analisadas pelo questionário de silhuetas dispostos de forma aleatória, observa-se uma menor prevalência de insatisfação em $68 \%$ das voluntárias (Figura 2).

Assim, o índice Kappa revelou uma concordância classificada como fraca entre as medidas $(0,46)$. Essa fraca concordância indica que adolescentes que apontaram insatisfação corporal em uma determinada forma de aplicação do questionário, não necessariamente foram classificadas como insatisfeitas na outra forma de aplicação do mesmo instrumento. Nesse sentido, a aplicação do questionário de silhuetas em adolescentes no seu formato original merece atenção; e a interpretação dos resultados deve ser analisada com cautela, pois, muitas vezes, os indivíduos classificados como insatisfeitos com o corpo podem, na verdade, não apresentar esse comportamento.

Esses resultados corroboram o estudo de Doll, Ball, e Willows ${ }^{15}$, no qual analisaram a disposição das silhuetas em três formatos diferentes para avaliação da imagem corporal em estudantes universitários, de ambos os sexos. Os resultados apontam diferenças significantes entres as três formas de apresentações das figuras, demonstrando a influência que esses instrumentos podem exercem na percepção corporal.

Apesar de ser uma importante ferramenta para avaliar a insatisfação com a imagem corporal, o método de imagens apresenta algumas limitações na sua metodologia. Alguns dos erros mais comuns cometidos por pesquisadores em relação a esse método são apontados por Thompson ${ }^{20}$. Dentre eles, destaca-se a problemática da escolha da imagem que mais se assemelha com a dimensão corporal do avaliado; assim, uma estratégia para lidar com essa dificuldade seria a inclusão de várias medidas 
corporais a fim de minimizar os erros de interpretações deturpadas.

Além disso, o instrumento deve ser desenvolvido ou testado em uma amostra similar aquela que se pretende avaliar. Muitas vezes, as silhuetas desenvolvidas para mulheres podem não apresentar os mesmos resultados em adolescentes. Assim, acredita-se que a falta de controle dessas variáveis possa influenciar na divergência entre as duas metodologias.

\section{Conclusão}

Conclui-se que as formas de aplicação da escala de silhuetas, crescente e aleatória, são discordantes. Além disso, a aplicação da forma crescente de silhuetas parece ter influenciado as adolescentes em uma opção distorcida da sua própria imagem corporal, sugerindo que o formato com que se aplicou essa escala influenciou a percepção da própria imagem corporal entre as adolescentes estudadas.

\section{Referências}

1. Storvoll EE, Strandbu A, Wichstrom L. A crosssectional study of changes in Norwegian adolescents body image from 1992 to 2002. Body Image. 2005;2:5-18.

2. Eisenberg ME, Neumark- Sztainer D, Paxton SJ. Fiveyear change in body satisfaction among adolescents. J Psychosom Res. 2006;61:521-7.

3. Stice E, Bearman SK. Body image and eating disturbances prospectively predict increases in depressive symptoms in adolescent girls: a growth curve analysis. Developmental Psychology. 2001:37:597-607.

4. Knauss C, Paxton SJ, Alsaker FD. Relationships amongst body dissatisfaction, internalization of the media body ideal and perceived pressure from media in adolescent girls and boys. Body Image. 2007;7:353-60.
5. Durkin Paxton SJ. Predictors of vulnerability to reduce body image satisfaction and psychological wellbeing in response to exposure to idealized female media images in adolescent girls. J Psychosom Res. 2002;53:995-1005.

6. Conti MA. Os aspectos que compõem o conceito de imagem corporal pela ótica do adolescente. Rev Bras Crescimento Desenvolv Hum. 2008;18:240-53.

7. Stunkard A J, Sorensen T, Schalsinger F. Use of the Danish adoption register for the study of obesity and thinness. Genetics of Neurological and Psychiatric Disorders. 1983;60:115-20.

8. Fallon AE, Rozin P. Sex differences in perceptions of desirable body shape. J Abnorm Psychol. 1985;94:102-5.

9. Collins ME. Body figure perceptions and preferences among preadolescent children. Int J Eat Disord. 1991;10:199-208.

10. Rand CS, Wright BA. Continuity and change in the evaluation of ideal and acceptable body sizes across a wide age span. Int J Eat Disord. 2000;28:90-100.

11. Scagliusi FB, Avarenga M, Polacow VO, Cordás TA, Queiroz GKO, Coelho D et al. Concurrent and discriminant validity of the Stuhnkard's figure rating scale adapted into Portuguese. Appetite. 2006;47:77-82

12. Bulik CM, Wade TD, Heath AC, Martin NG, Stunkard AJ, Eaves LJ. Relating body mass index to figural stimuli: population based normative data for Caucasian. Int Jf Obes. 2001;25 (10):1517-25.

13. Mueller WH, Joo SK, Schull, WJ. Alternative measurements of obesity: accuracy of body silhouettes and reported weights and heights in a Mexican American sample. Int J Obes.1985;9(3):193200.

14. Gardner RRM, Friedman BN, Jackson NA. Methodological concerns when using silhouettes to measure body image. Percept Mot Skills. 1998;86:387-95.

15. Doll M, Ball GDC, Willows ND. Rating of figures used for body image assessment varies depending on the method of figure presentation. Int J Eat Disord. 2004;35:109-14.

16. Gordon CC, Chumlea WC, Roche AF. Stature, recumbent length, and weight. In: Lohman TG, Roche AF, Martoreli R, editors. Anthropometric standardizing reference manual. Champaign, Illinois: Human Kinetics Books; 1988. p. 3-8. 
17. Cole TJ, Bellizzi MC, Flegal KM, Dietz WH. Establishing a standard definition for children overweight and obesity worldwide: international survey. British Medical Journal. 2000;302:1240-3.

18. Davis DS, Sbrocco T, Williams J. Understanding body image in African American and Caucasian first- graders: a partnership with the YMCA. Program Community Health Partnersh. 2009;3(4):277-86.
19. Sato A, Le DS, Tran MH, Kaneda M, Murai E, Kamiyama H, Oota Y, Yamamoto S. Study on factor of body image in Japanese and Vietnamese adolescent. J Nutr Sci Vitaminol. 2008;54(2):169-75.

20. Thompson JK. The (mis) measurement of body image: ten strategies to improve assessment for applied and research purposes. Body Image. 2004;7-14. 